\title{
Non-functional duodenal neuroendocrine carcinoma: a rare cause of diabetes mellitus
}

\author{
Chad Bisambar', Andrew Collier ${ }^{1}$, Fraser Duthie ${ }^{2}$ and Carron Meney ${ }^{1}$ \\ ${ }^{1} \mathrm{NHS}$ Ayrshire and Arran, Ayr, UK and ${ }^{2} \mathrm{NHS}$ Greater Glasgow and Clyde, Glasgow, UK
}

Correspondence

should be addressed

to C Bisambar

Email

chadbisambarmd@gmail.

com

\section{Summary}

A 40-year-old Caucasian female presented with hyperglycaemia, polyuria, polydipsia and weight loss of $6 \mathrm{~kg}$ over a 1-month period. There was no personal or family history of malignancy or diabetes mellitus. On examination, she was jaundiced with pale mucous membranes and capillary glucose was $23.1 \mathrm{mmol} / \mathrm{L}$. Initial investigations showed iron deficiency anaemia and obstructive pattern of liver function tests. HbA1c was diagnostic of diabetes mellitus at $79 \mathrm{mmol} / \mathrm{mol}$. Malignancy was suspected and CT chest, abdomen and pelvis showed significant dilatation of intra- and extra-hepatic biliary tree including pancreatic duct, with periampullary $30 \mathrm{~mm}$ mass lesion projecting into lumen of duodenum. Enlarged nodes were seen around the superior mesenteric artery. This was confirmed on MRI liver. Fasting gut hormones were normal except for a mildly elevated somatostatin level. Chromogranin A was elevated at $78 \mathrm{pmol} / \mathrm{L}$ with normal chromogranin B. Duodenoscopy and biopsy showed possible tubovillous adenoma with low-grade dysplasia, but subsequent endoscopic ultrasound and biopsy revealed a grade 1, well differentiated neuroendocrine tumour. The patient was started on insulin, transfused to $\mathrm{Hb}>8 \mathrm{~g} / \mathrm{dL}$ and Whipple's pancreatico-duodenectomy was undertaken. This showed a well-differentiated neuroendocrine carcinoma arising in duodenum (Grade G1 with Ki67: 0.5\%), with areas of chronic pancreatitis and preservation of pancreatic islet cells. There was complete resolution of diabetes post Whipple's procedure and patient was able to come of insulin treatment. Her last HBA1C was $31 \mathrm{mmol} / \mathrm{mol}, 4$ months post tumour resection.

\section{Learning points:}

- Diabetes mellitus and malignancy can be related.

- A high index of suspicion is needed when diabetes mellitus presents atypically.

- Non-functional neuroendocrine tumours can present with diabetes mellitus.

\section{Background}

Non-functional duodenal neuroendocrine tumours can present with hyperglycaemia and cause diabetes. This is in the absence of gluconeogenic hormones such as somatostatin and glucagon.

\section{Case presentation}

A previously well 40-year-old Caucasian female was admitted with hyperglycaemia, polyuria, polydipsia and weight loss of $6 \mathrm{~kg}$ over a 1-month period.
She denied any respiratory symptoms or night sweats. She did not suffer from nausea, vomiting, diarrhoea, constipation or any symptoms of gastrointestinal bleeding.

There was no personal or family history of malignancy or diabetes mellitus. She denied alcohol, cigarette and illicit drug use. She took no prescription or over-thecounter medication and had no allergies.

On examination, she was jaundiced with pale mucous membranes. The rest of systemic examination was 
normal. Capillary glucose was found to be $23.1 \mathrm{mmol} / \mathrm{L}$. Her weight was $98 \mathrm{~kg}$.

\section{Investigations}

Investigations showed microcytic anaemia with haemoglobin $(\mathrm{Hb})$ of $6.4 \mathrm{~g} / \mathrm{dL}$, and the blood film was consistent with iron deficiency anaemia. This most likely was the result of occult upper gastrointestinal bleeding. The laboratory glucose was elevated at $21.8 \mathrm{mmol} / \mathrm{L}$ with glycosylated haemoglobin (HBA1c) of $79 \mathrm{mmol} /$ mol representing a new diagnosis of diabetes mellitus. The urinary albumin to creatinine ratio was also elevated at 5.4 .

Liver function tests showed an obstructive picture with total bilirubin 48 (3-21 U/L), alkaline phosphatase (ALP) of 687 (33-144U/L), aspartate transaminase (AST) of 969 (10-45 U/L) and alanine transaminase (ALT) of 117 (5-55 U/L). Prothrombin time and albumin were normal. Liver screen including AFP, alpha 1-antitrypsin, copper studies, anti-smooth muscle antibody, anti-mitochondrial antibody and hepatitis screen were all normal. Renal function tests were also normal.

Chest X-ray on admission showed no abnormalities. Gastrointestinal malignancy was suspected and subsequent CT chest, abdomen, pelvis showed significant dilatation of intra- and extra-hepatic biliary tree including the pancreatic duct, and a periampullary $30 \mathrm{~mm}$ mass lesion projecting into lumen of duodenum. Enlarged lymph nodes were seen around superior mesenteric artery but no distal metastases were found (Figs 1 and 2).

This was confirmed on MRI liver (Figs 3 and 4).

The case was discussed at the local upper gastrointestinal multi-disciplinary team (MDT) meeting and the outcome was that the findings were in keeping with a neuroendocrine tumour (NET) given the location and possible hormonal hypersecretion contributing to hyperglycaemia.

Fasting gut hormones were subsequently done showing vasoactive intestinal peptide (VIP) of 4 (<30 pmol/L), pancreatic polypeptide of $12(<300 \mathrm{pmol} / \mathrm{L})$, gastrin of $8(<40 \mathrm{pmol} / \mathrm{L})$, glucagon of $14(0-50 \mathrm{pmol} / \mathrm{L})$ and somatostatin of $174(0-150 \mathrm{pmol} / \mathrm{L})$.

Chromogranin A was mildly elevated at 78 (0-60 pmol/L) and chromogranin $\mathrm{B}$ was normal at 49 (0-150 pmol/L).

A subsequent screen for MEN-1 syndrome including prolactin, calcium and PTH was normal.

The patient subsequently underwent duodenoscopy, which showed a flat velvet-like lesion in anterior wall

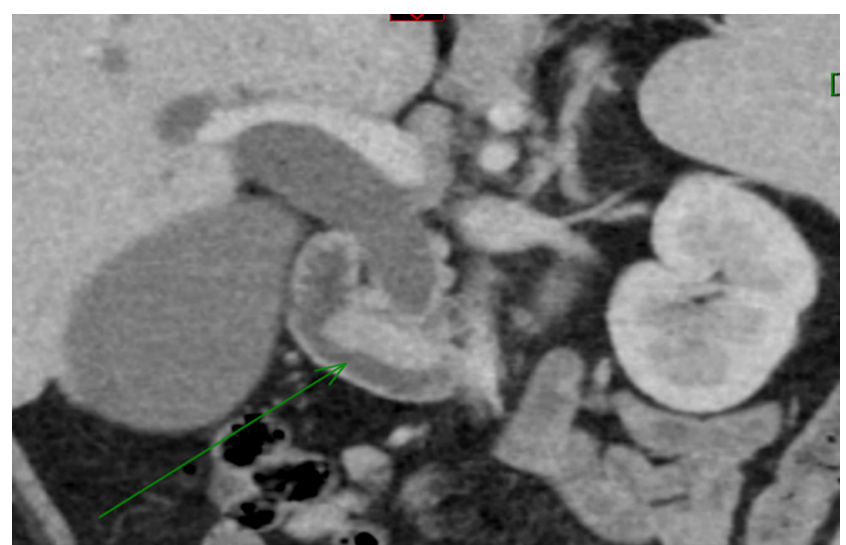

Figure 1

CT scan showing periampullary $30 \mathrm{~mm}$ mass lesion projecting into lumen of duodenum (coronal section).

of 2nd part of duodenum around the ampulla (Fig. 5). The biopsy was reported as tubovillous adenoma with low-grade dysplasia with no evidence of neuroendocrine tumour.

Endoscopic ultrasound was then performed and showed a mass in medial wall of the duodenum and a suspicious node over superior mesenteric artery. The fineneedle biopsy of duodenal wall lesion and lymph node were in keeping with grade 1 , well-differentiated duodenal neuroendocrine tumour (d-NET).

Furthermore, an octreotide whole-body scan and SPECT CT showed no uptake.

\section{Treatment}

The patient was started on insulin 30\% soluble insulin mixed with 70\% isophane insulin (humulin M3)

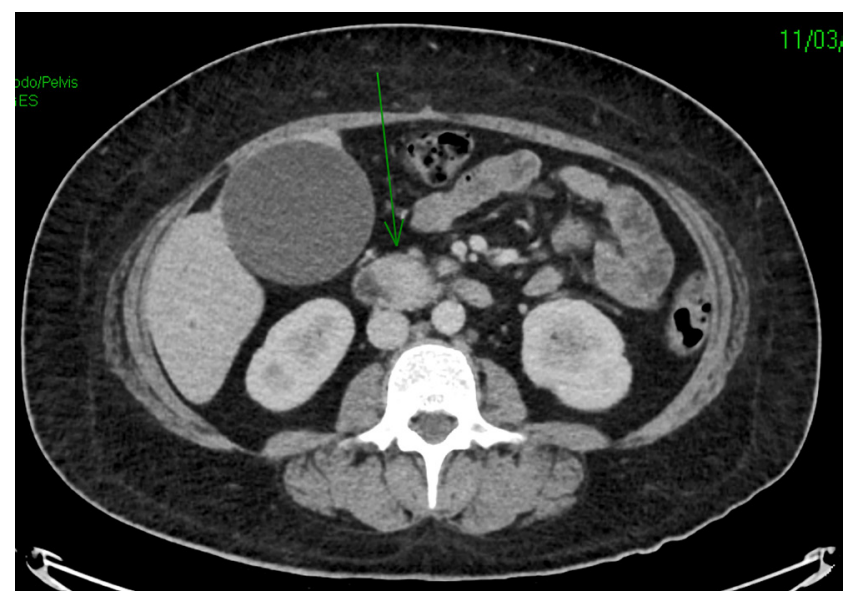

Figure 2

Axial view of the periampullary tumour on CT scan. 


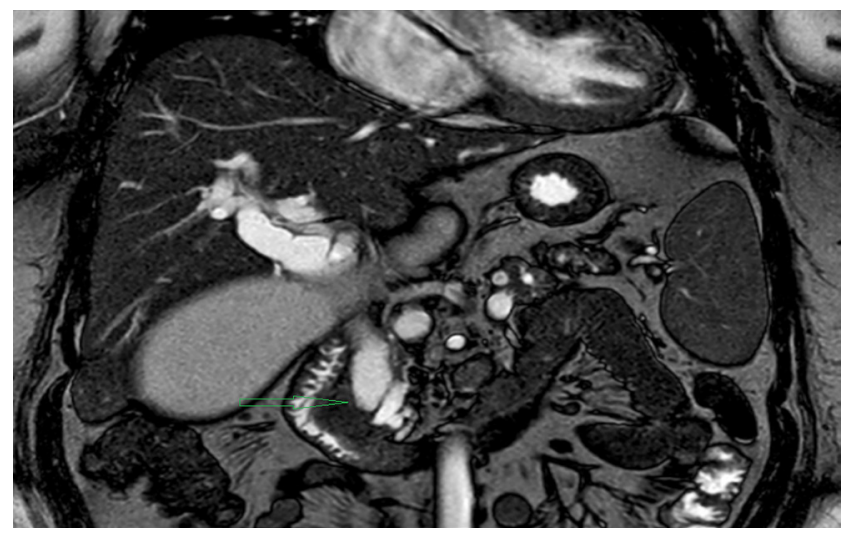

Figure 3

MRI liver confirming periampullary lesion found on CT scan (coronal view).

subcutaneously twice daily (6-14 units at breakfast, 6-12 units at teatime) and transfused with partially packed red blood cells to $\mathrm{Hb}>8 \mathrm{~g} / \mathrm{dL}$. The HbA1c 3 months after commencement of insulin was $39 \mathrm{mmol} / \mathrm{mol}$.

After further MDT discussion between endocrinology, oncology, radiology and surgical teams, Whipple's pancreatic-duodenectomy was planned and carried out. This showed a well-differentiated neuroendocrine carcinoma arising in the duodenum (R0 pT3 pN1). The tumour was classified as grade G1 with Ki67: 0.5\% (Fig. 6). Venous invasion was present and there was involvement of 4 of 17 lymph nodes.

Histological staining is as shown (Figs 7 and 8).

Preservation of islet cells was seen within areas of pancreatitis (Fig. 9).

Immunohistochemical staining of the tumour was not done by the pathology team as clinically the tumour

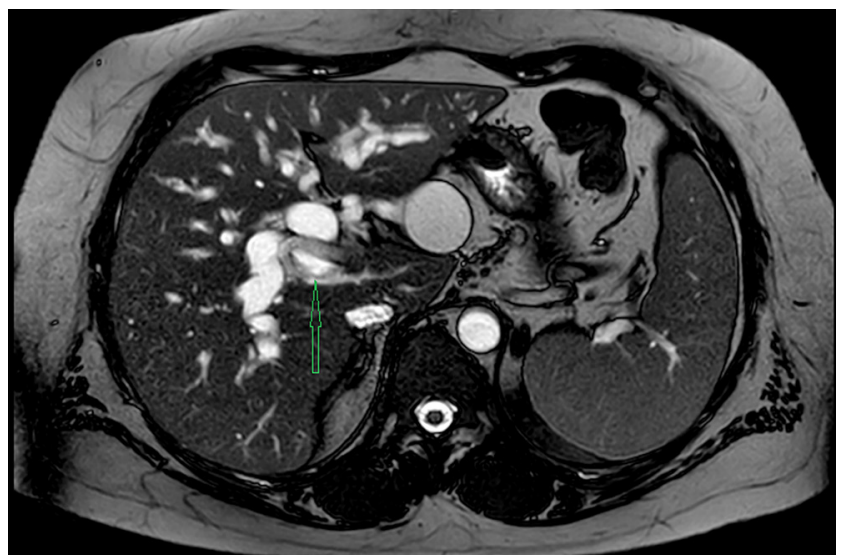

Figure 4

MRI showing axial view of periampullary tumour.

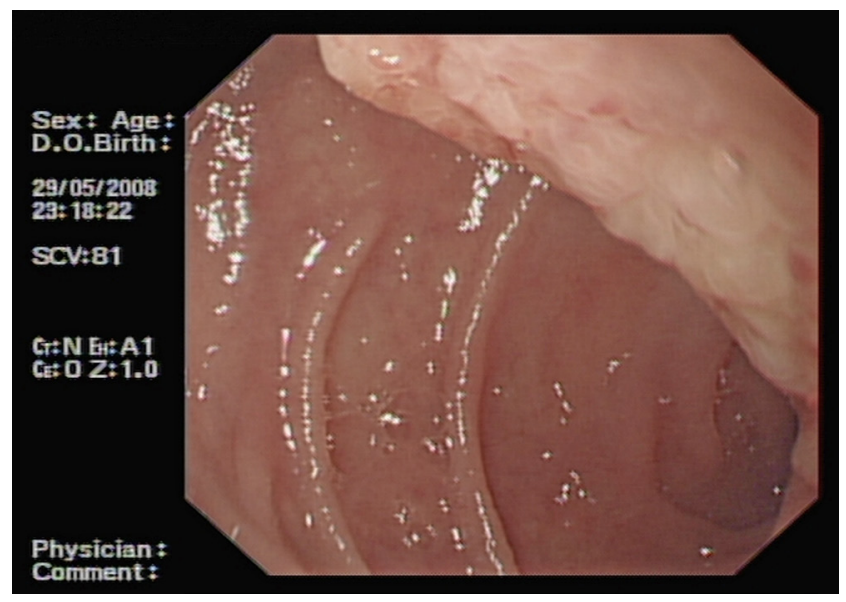

Figure 5

Flat velvet-like lesion in anterior wall of 2 nd part of duodenum around the ampulla.

was non-functional and immunohistochemical findings do not usually correlate with serum hormonal levels.

\section{Outcome and follow-up}

The patient did not require insulin postoperatively and HbA1c was $31 \mathrm{mmol} / \mathrm{mol} 4$ months after surgery. There was complete resolution of diabetes post Whipple's procedure and the patient is now off insulin.

Follow-up CT scan chest/abdomen/pelvis is pending, and chromogranin A was normal at $32 \mathrm{pmol} / \mathrm{L}, 6$ months after tumour resection.

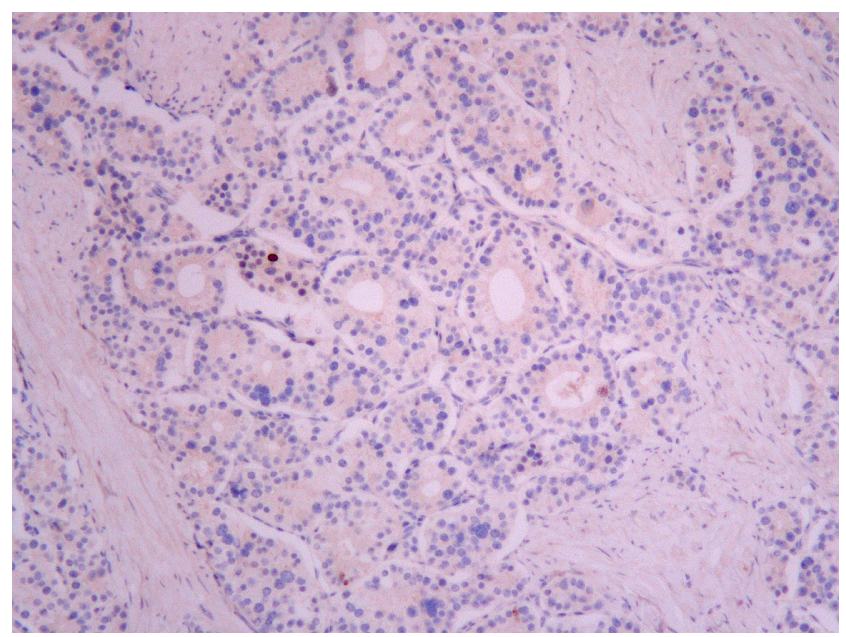

Figure 6

Medium power Ki67 image showing staining within the nucleus of a tumour cell. 


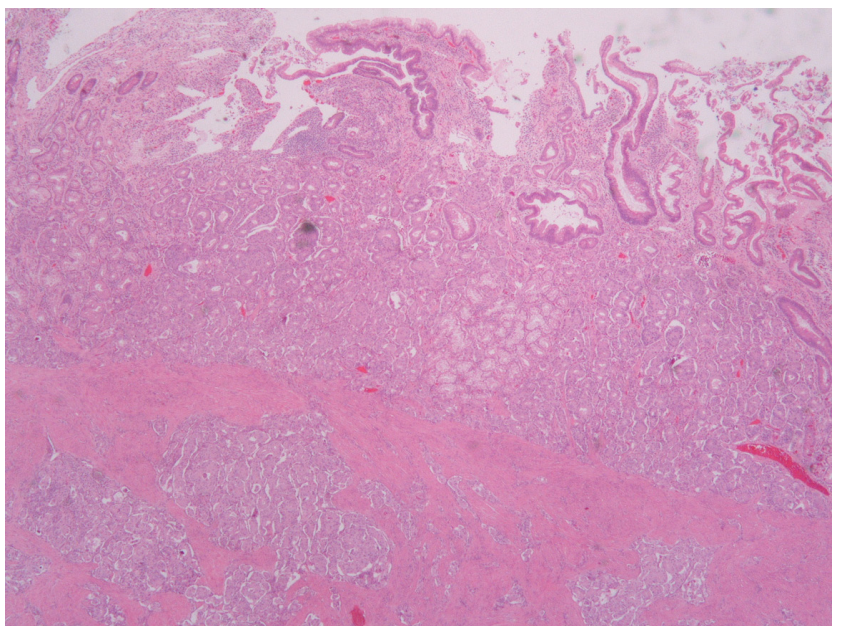

Figure 7

Low-power haematoxylin and eosin image showing tumour arising in the mucosa, replacing the submucosa and infiltrating the muscularis propria.

\section{Discussion}

Duodenal neuroendocrine tumours (d-NETs) represent less than 3\% of all duodenal tumours. Approximately 40-60\% of patients at the time of diagnosis have metastases to regional lymph nodes and 10\% have liver metastases (1). The majority of d-NETs are non-functional, but $10 \%$ are gastrinomas and less than $4 \%$ present with typical carcinoid syndrome.

On the basis of gene expression profiles, NETs of the duodenum are considered a distinct entity from tumours of jejunum and ileum $(2,3)$. Although primary d-NETs are rare, slow-growing neoplasms with indolent

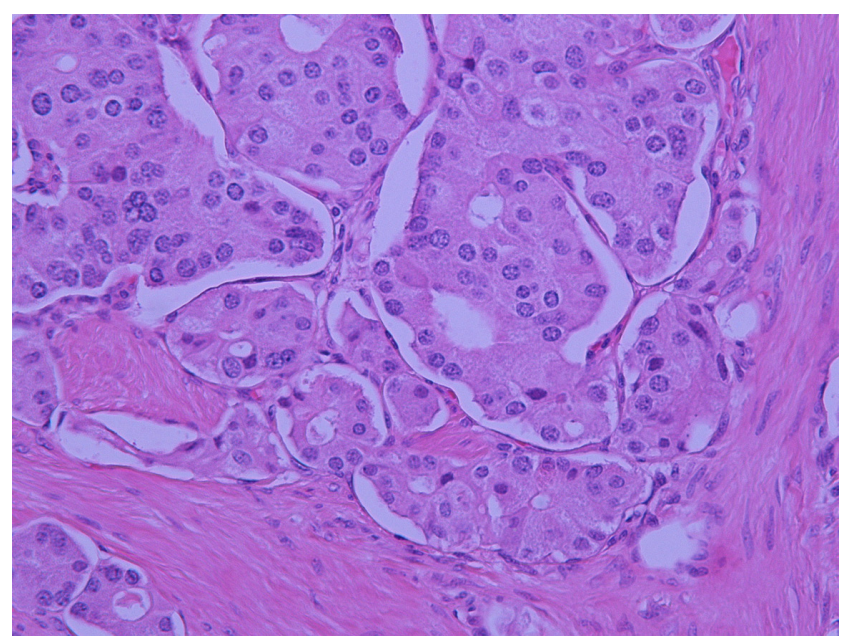

Figure 8

High-power haemotoxylin and eosin image showing the typical appearance of a neuroendocrine tumour with cells are arranged in packets with round nuclei and granular cytoplasm.

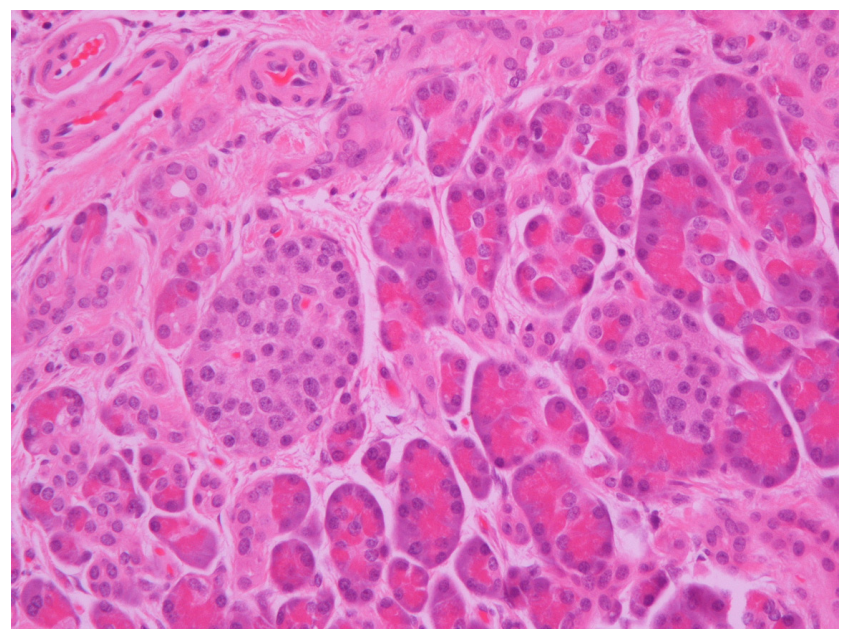

Figure 9

Residual islet of Langerhans are seen within areas of chronic pancreatitis.

clinical behaviour, they can be potentially malignant (4). These tumours present commonly with abdominal pain, upper gastrointestinal bleed, constipation, anaemia or jaundice (5).

Glucagon or somatostatin producing d-NET's can also present with hyperglycaemia or frank diabetes mellitus. In our case, however, the mildly elevated somatostatin level was not significant and glucagon levels were normal. Clinically, the tumour was non-functional.

Neuroendocrine tumours with pancreatic duct obstruction have been reported to cause hyperglycaemia and cause diabetes. This is in the absence of gluconeogenic hormones such as somatostatin and glucagon.

Murray et al. (6) in 2016 reported a similar case in a middle aged, black, North American male who presented with newly diagnosed diabetes and was found to have a non-functional d-NET obstructing the biliary and pancreatic ducts. There was persistence of diabetes following surgical removal. Another study by Koshimizu (7) in 2012 describes aggravation of existing diabetes in a 65-year-old man by a non-functional pancreatic NET obstructing the main pancreatic duct, causing chronic pancreatitis and beta cell damage. After tumour removal, the patient still required insulin treatment.

Very interestingly in our case, not only were the islet of Langerhans preserved, but the patient had developed diabetes mellitus, which was cured postoperatively. This raises the question as to whether our patient had developed diabetes due to an undetected gluconeogenic hormone being produced by the tumour more likely than due to an obstructive component or chronic pancreatitis. 
Very importantly, this case highlights that diabetes mellitus and malignancy can be related and that a high index of suspicion is needed when diabetes presents atypically.

\section{Declaration of interest}

The authors declare that there is no conflict of interest that could be perceived as prejudicing the impartiality of the research reported.

\section{Funding}

The research did not receive any specific grant from funding agency in the public, commercial or not-for-profit sector.

\section{Patient consent}

Written consent has been obtained from the patient for publication of this article and accompanying images.

\section{Author contribution statement}

Prof. Andrew Collier: Lead consultant responsible for management of patient. Dr Fraser Duthie: Lead pathologist. Dr Carron Meney: Assisted with data collection and case report submission.

\section{References}

1 O'Toole D, Delle Fave G \& Jensen RT. Gastric and duodenal neuroendocrine tumours. Best Practice and Research: Clinical Gastroenterology 201226 719-735. (https://doi.org/10.1016/j. bpg.2013.01.002)

2 Klimstra DS, Modlin IR, Coppola D, Lloyd RV \& Suster S. The pathologic classification of neuroendocrine tumors: a review of nomenclature, grading, and staging systems. Pancreas 201039 707-712. (https://doi.org/10.1097/MPA.0b013e3181ec124e)

3 Modlin I, Lye K \& Kidd M. A 5-decade analysis of 13715 carcinoid tumors. Cancer 200397 934-959. (https://doi.org/10.1002/ cncr.11105)

4 Kim SH, Park CH, Ki HS, Jun CH, Park SY, Kim HS, Choi SK \& Rew JS. Endoscopic treatment of duodenal neuroendocrine tumors. Clinical Endoscopy 201346 656-661. (https://doi.org/10.5946/ ce.2013.46.6.656)

5 Hoffmann KM, Furukawa M \& Jensen RT. Duodenal neuroendocrine tumors: classification, functional syndromes, diagnosis and medical treatment. Best Practice and Research Clinical Gastroenterology 200519 675-697. (https://doi.org/10.1016/j.bpg.2005.05.009)

6 Murray L, Haley C, Berry-Cabán CS \& Toledo A. Duodenal neuroendocrine tumor and the onset of severe diabetes mellitus in a US veteran. SAGE Open Medical Case Reports 4 1-3. (https://doi.org/10 $.1177 / 2050313 \times 15624530)$

7 Koshimizu H, Omori H \& Funase Y. Pancreatic nonfunctioning neuroendocrine tumor with the main pancreatic duct obstruction presenting as excessive hyperglycemia: a case report and review of the literature. Pancreas 201241 160-163. (https://doi.org/10.1097/ MPA.0b013e318221c4c1)

Received in final form 2 July 2018

Accepted 7 August 2018 